\title{
Custo do capital próprio como taxa de desconto na avaliação de empresas no Brasil: evidências entre a teoria e a prática de mercado
}

\author{
Cost of equity as discount rate in firms' evaluation in Brazil: evidence between theory and \\ market practice
}

\author{
Ludmila Lopes Cabral \\ $\underline{\text { lud_cabral@yahoo.com.br }}$ \\ Universidade Federal de Goiás \\ Camila Araujo Machado \\ ufg_face_camila@yahoo.com.br
}

Universidade Federal de Goiás

\author{
Moisés Ferreira da Cunha \\ mfccunha@ig.com.br \\ Universidade Federal de Goiás \\ Ilírio José Rech \\ ilirio@terra.com.br
}

Universidade Federal de Uberlândia

\section{RESUMO}

A avaliação de empresa é potencialmente praticada e discutida pelos teóricos. Dentre os métodos de avaliação, o mais utilizado é o Fluxo de Caixa Descontado (FCD), cujo sucesso dependerá da correta determinação da taxa de desconto. Neste método, a mensuração do custo do capital próprio (Ke) é uma das etapas mais relevantes, pela maior subjetividade envolvida. Em mercados emergentes torna-se mais desafiador a mensuração desta taxa de desconto. Este artigo tem o objetivo de verificar se no Brasil existe aderência entre teoria e a prática do mercado quanto à estimação do Ke para valoração de empresas pelo FCD. A hipótese levantada é a de que as práticas do mercado brasileiro na determinação do Ke para avaliação de empresas pelo FCD estão condizentes com a teoria de finanças. Para verificar a hipótese, realizou-se uma pesquisa documental através da investigação dos Laudos de Avaliação das Companhias apresentados como requisito às ofertas públicas de aquisição de ações (OPA) registrados na Comissão de Valores Mobiliários (CVM) no período de 2005 a 2012. A amostra perfez 96 laudos os quais mensuraram o valor da entidade pelo FCD. A análise de resultados comprovou a aderência dos métodos utilizados pelos praticantes brasileiros e a literatura de finanças sobre o tema, levando a não rejeição da hipótese proposta inicialmente. Desta forma, as evidências encontradas neste estudo contribuem com os estudiosos, avaliadores e demais interessados no assunto e, atribui-se maior credibilidade às informações geradas pelas avaliações de empresas no Brasil, qualitativamente.

Palavras-chave: Avaliação de Empresas, Fluxo de Caixa Descontado, Custo do Capital Próprio.

\section{ABSTRACT}

Firm's evaluation is potentially practiced and discussed by theoretical. Among the evaluation's methods the most used is the Discounted Cash Flow (DCF), which has success whether the right discount rate is determined. In this method, the measurement of the cost of equity $(\mathrm{Ke})$ is one of the most relevant stages, because of the subjectivity involved. In emergent markets, the measurement of this discount rate becomes harder. The aim of this paper is to verify whether has an adherence between theory and market practice in Brazil about the measurement of Ke in the scope of firms' appreciation by DCF. The raised hypothesis is that the brazilian market 
practices of the Ke's determination to the firms' evaluation by DCF are consistent with the financial theory. To verify this hypothesis, a documental research among the Firms' Evaluation Reports, which are presented as Initial Public Offering (IPO) requirement and were registered in Comissão de Valores Mobiliários (CVM) in the years 2005 to 2012, has been done. The sample has found 96 reports that measure the firms' value by the DCF. The results' analysis proven the adherence of the methods used by brazilians practitioners and the finance literature about this theme, what lead a non-rejection of the initially purposed hypothesis. Therefore, the evidence found in this paper contribute with researchers, appraisers and who else that have interest in this subject and give more credibility for the information generated by firms' evaluation in Brazil, qualitatively.

Keywords: Firms' Evaluation, Discounted Cash Flow, Cost of Equity.

Artigo recebido em: 25.11.2013; Aceito em: 15.02.2014

\section{Introdução}

Em um cenário globalizado observa-se que avaliação de empresas tornou-se uma temática significativamente discutida pelo mercado, pela academia e pela imprensa. No Brasil essa discussão se intensificou nos anos 90, quando novos condicionantes foram apresentados à economia brasileira. Atualmente, empresas são avaliadas com vistas aos mais diferentes propósitos, entre eles: fusão, cisão e incorporação; abertura do capital; mensuração da capacidade dos gestores em gerar riqueza ao acionista; e, ainda, para disputas judiciais.

O processo de avaliação de empresa consiste na estimativa do valor que represente a sua potencialidade econômica através da avaliação conjunta de três componentes principais os quais interagem entre si: ativos, capitais de terceiros e capital próprio da empresa. Dentre os métodos de avaliação, os mais utilizados são baseados nos valores projetados de fluxos de caixa descontados a valor presente por uma taxa que reflita o risco inerente, conhecidos como Fluxo de Caixa Descontado - FCD.

Os métodos que se baseiam no FCD são os mais populares, uma vez que, segundo Fernández (2007), são os conceitualmente "corretos". A importância do FCD, para Assaf Neto e Lima (2009, p.740), se deve ao fato de esse método apresentar maior rigor técnico e conceitual. De acordo com os mesmos autores $(2009$, p. 752$)$, os fluxos de caixa utilizados na avaliação de empresas é o Fluxo de Caixa Livrei, subdividido em: Fluxo de Caixa Livre da Empresa - FCLE, geralmente mais utilizado, e Fluxo de Caixa Livre do Acionista - FCLA. O FCLE usualmente é descontado pelo custo médio ponderado de capital (WACC ${ }^{\mathrm{ii}}$ ) e os FCLA pelo custo de oportunidade do capital próprio (Ke). O Ke é geralmente estimado pelo modelo de precificação de ativos mais difundido mundialmente, o CAPM, cuja prática de sua utilização é reforçada por Copeland, Koller e Murrin (2002, p. 230), pois, para eles, a teoria envolvida no modelo CAPM não pode ser substituída já que ainda não se sabe de outra teoria melhor.

Fernández (2007) chama a atenção para o fato de que a taxa de desconto é uma das tarefas mais importantes no processo de avaliação de empresas, por considerar os riscos e a volatilidade histórica. A subjetividade do processo de avaliação pelo FCD, para Garrán e Martelanc (2007), concentra-se na estimação da magnitude dos fluxos de caixa futuros e na taxa de desconto utilizada. Além da subjetividade, outro fator que potencializa o interesse sobre o assunto é o fato de que as metodologias aplicadas na mensuração das taxas de desconto e na valoração de empresas são originadas nos mercados desenvolvidos. 
Segundo Assaf Neto, Lima e Araújo (2008) o correto conhecimento do custo de capital é essencial para o processo de análise e tomada de decisões financeiras, porém existem dificuldades no cálculo do WACC, principalmente na determinação do custo do capital próprio (Ke). Martins et al (2006) também ressaltam que a mensuração do Ke é uma das etapas mais relevantes no processo de avaliação de empresas já que os modelos praticados com esta finalidade mostram-se bastante sensíveis às alterações nas taxas de desconto, e que, normalmente essa taxa $(\mathrm{Ke})$ integra esses modelos como um de seus principais componentes, a exemplo do custo médio ponderado de capital (WACC).

Sabendo que os modelos de avaliação realizados através do FCD são os preconizados pela teoria de finanças, e que dentre esses métodos, existem duas abordagens principais (FCLE e FCLA) as quais integram o Ke; cientes da existência de diferentes concepções teóricas sobre o assunto, e das muitas variáveis envolvidas no cálculo dessa taxa, surge a necessidade de se responder a seguinte indagação: as práticas do mercado brasileiro para a determinação do Ke na avaliação de empresas pelo método do Fluxo de Caixa Descontado estão condizentes com as teorias preconizadas na academia?

Uma vez que a avaliação de empresas é tão difundida, utilizada de forma recorrente por diversos usuários e com diferentes propósitos, e que fundamentadas nesta estimativa são tomadas decisões importantes, as quais são condicionadas à credibilidade dada pelos usuários às informações provenientes destas avaliações, segue a hipótese de trabalho desta pesquisa $(\mathrm{H})$ :

\section{H: As práticas do mercado brasileiro na determinação do Ke para avaliação de empresas pelo FCD estão condizentes com a teoria de finanças.}

A pesquisa justifica-se por servir de fonte de informações para os profissionais atuantes na avaliação de empresas e demais interessados pelo tema, clarificar as teorias para mensuração do custo de capital próprio na avaliação de empresas, buscando ainda atenuar a assimetria. A pesquisa também tem o propósito de fornecer um feedback ao mercado e à comunidade acadêmica sobre as práticas na avaliação de empresas. O Objetivo deste estudo é verificar se no Brasil existe aderência entre a academia e as práticas do mercado no processo de estimação do Ke com a finalidade de Oferta Pública de Aquisição de Ações no período entre 2005 a 2012.

Este artigo encontra-se estruturado em mais quatro seções. Na segunda seção apresenta-se o referencial teórico sobre o custo do capital próprio. Posteriormente, clarifica-se a metodologia aplicada a fim de se atingir os desígnios da pesquisa. A quarta seção traz a apresentação e análise dos resultados oriundos da investigação dos laudos de ofertas públicas de ações - OPAs relacionados com a teoria. Por fim, são explanadas considerações a respeito da pesquisa.

\section{O CUSTO DO CAPITAL PRÓPRIO - Ke}

\subsection{Modelos Aplicáveis no Cálculo do Custo de Capital Próprio}

Lima (2007) destaca quatro modelos de estimação desta variável: (i) o Modelo de Gordon; (ii) o Modelo Ohlson-Juettner (OJ); (iii) o Arbitrage Princing Model (APM) e (iv) o Capital Asset Pricing Model (CAPM). No modelo de Gordon (Expressão 1), ou abordagem dos descontos dos fluxos de dividendos, o retorno do acionista corresponde ao valor presente dos dividendos esperados somados à sua taxa de crescimento constante. 
Onde:

$$
\mathrm{Ke}=\frac{\mathrm{dps}}{\mathrm{P}_{0}}+\mathrm{g}
$$

$\mathrm{Ke}=$ custo de capital próprio;

dps = dividendo por ação;

$\mathrm{P}_{0}=$ preço por ação na data atual;

$\mathrm{g}=$ taxa de crescimento constante dos dividendos.

Martins et al (2006) descrevem o modelo desenvolvido em 2005 por Ohlson e Juettner-Nauroth - OJ, no qual o retorno do acionista é encontrado pela expressão 2:

$$
\mathrm{Ke}=\frac{1}{2}\left(\gamma-1+\frac{\mathrm{dps}_{1}}{\mathrm{P}_{0}}\right)+\sqrt{\frac{1}{2}\left(\gamma-1+\frac{\mathrm{dps}_{1}}{\mathrm{P}_{0}}\right)^{2}+\frac{\mathrm{eps}_{1}}{\mathrm{P}_{0}} \times\left(\frac{\Delta \mathrm{eps}_{2}}{\mathrm{eps}_{1}}-(\gamma-1)\right)}
$$

Sendo:

$\mathrm{Ke}=$ custo de capital próprio;

$\gamma=$ taxa de crescimento constante esperada dos dividendos;

dps = dividendo por ação no final do período 1 ;

$\mathrm{P}_{0}=$ preço por ação na data atual;

eps ${ }_{1}=$ lucro por ação esperado ao final do período 1 ;

$\Delta \mathrm{eps}_{2} / \mathrm{eps}_{1}=$ crescimento esperado do lucro do período 1 em relação ao período 2 .

Copeland, Koller e Murrin (2002, p. 230) relatam que evidências empíricas sugerem que o APM explique os retornos previstos melhor do que o CAPM. O custo do capital próprio, no APM, é assim definido:

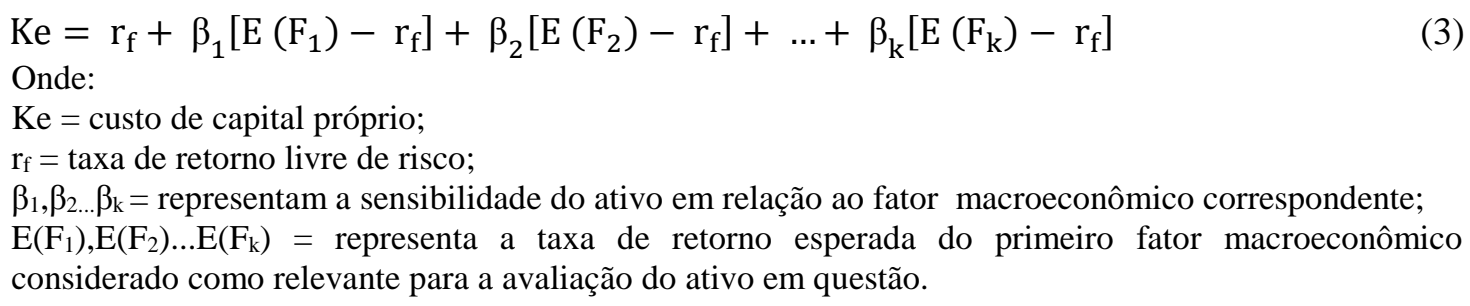

O APM, teoricamente, permite a modelagem individual e explícita de componentes típicos de risco-país (exemplo: inflação e risco cambial) e, portanto, poderia ser um bom substituto para o CAPM em mercados emergentes. Ainda assim, o analista não é confrontado com uma série de dados macroeconômicos os quais são normalmente incompletos, extremamente voláteis, tornando o uso deste modelo impraticável (PEREIRO, 2006).

De acordo com Garrán e Martelanc (2007), 60,22\% dos profissionais brasileiros envolvidos com a atividade de avaliação de ativos em diferentes setores e com diferentes fins, entre os anos de 2002 e 2006, utilizaram-se do CAPM para determinação do Ke no método do FCD; enquanto 26,88\% utilizaram o Método de Prêmios de Risco (Build-up Models), representado pela fórmula abaixo:

\footnotetext{
$\mathrm{Ke}=\mathrm{Rf}+\mathrm{PRm}+\mathrm{PRp}+\mathrm{PRe}$

Onde:

$\mathrm{Ke}=$ custo de capital próprio;

$\mathrm{Rf}=$ retorno do ativo livre de risco;

$\mathrm{PRm}=$ prêmio de risco de mercado;

$\mathrm{PRp}=$ prêmio risco de porte;

$\mathrm{PRe}=$ prêmio risco específico da empresa
} 
Na estimativa do custo do capital próprio, dar-se-á ênfase neste trabalho ao modelo CAPM, justificada pela superioridade teórica do modelo e por ser amplamente difundido entre os praticantes do mercado.

\subsection{Capital Asset Pricing Model - CAPM}

Dentre os modelos de cálculo do custo do capital próprio o mais utilizado é o CAPM, uma vez que seus princípios metodológicos não foram superados por qualquer outro modelo (COPELAND; KOLLER; MURRIN, 2002, p. 230; GARRÁN e MARTELANC, 2007). O CAPM postula que tal custo de oportunidade seja igual ao retorno sobre os títulos livres de risco mais o risco sistêmico da empresa multiplicado pelo ágio (prêmio) pelo risco no mercado. Os parâmetros que compõem tradicionalmente o CAPM estão demonstrados na expressão 5:

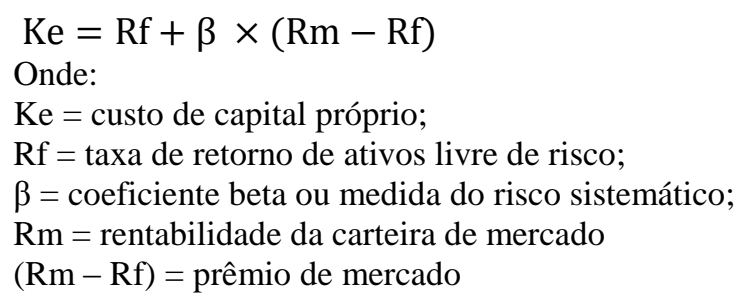

O CAPM possui como premissas norteadoras a eficiência do mercado e a diversificação, na qual o investidor possui uma carteira diversificada no mercado. Pereiro (2006) pondera que a existência desta eficiência em países emergentes é bastante discutível uma vez que estes possuem mercados pequenos, concentrados e sujeitos à manipulação. Perante esta crítica, Assaf Neto (2003) propõe a adoção do CAPM no Brasil através da utilização de benchmark de uma economia mais estável, como a dos EUA, ajustado pela incorporação do risco-país ao modelo, com o propósito de capturar riscos peculiares ao país.

\subsubsection{Taxa livre de risco - $\mathrm{Rf}$}

Um dos pressupostos do CAPM é a existência de uma taxa que os investidores podem obter em investimentos livres de risco. O modelo estima retornos esperados sobre investimentos arriscados através de sua relação com essa taxa livre de risco. Segundo Damodaran (2009, p.160), um ativo é livre de risco quando tal ativo não possua risco de inadimplência (default risk) nem risco de reinvestimento, o qual decorre, por exemplo, de diferenças entre os prazos do título e dos fluxos de caixa avaliados.

Normalmente os títulos livres de risco são os emitidos pelo governo. A taxa do Sistema Especial de Liquidação e Custódia de Títulos Públicos (Selic) é o parâmetro para ativo livre de risco no mercado brasileiro. Para Martins et al (2006) os títulos brasileiros não podem ser considerados como livres de risco, principalmente pelo fato de o governo ter decretado moratória duas vezes na história.

Diante das características brasileiras, Assaf Neto, Lima e Araújo (2008) sugerem que a taxa livre de risco utilizada no cálculo do Ke seja mensurada tendo-se como referência as taxas de juros pagas pelos melhores títulos de dívida do mundo, destacando-se os papéis emitidos pelo Tesouro do Governo dos Estados Unidos.

Copeland, Koller e Murrin (2002, p.220) apontam três opções de taxas livre de risco nos Estados Unidos: taxa das Letras do Tesouro de curto prazo (Treasury bills ou T-bills); taxa dos títulos 
do Tesouro (Treasury bonds ou T-bonds) de 10 anos; e T-bonds de 30 anos. Os autores recomendam a utilização da taxa $T$-bonds de 10 anos, entre outros motivos por se aproximar mais da duração dos fluxos de caixa projetados na fase explícita de projeção e ser menos suscetível a mudanças inesperadas de inflação (comparativamente aos T-bonds de 30 anos). É relevante a observância da coerência entre a taxa livre de risco e os fluxos de caixa estimados. A Rf deve ser estimada na mesma moeda na qual os fluxos de caixa da empresa são estimados, ou seja, se esses fluxos forem estimados em dólares americanos nominais, a taxa será a do bônus do Tesouro americano (DAMODARAN, 2009, p.161).

\subsubsection{Beta - $\beta$}

No modelo CAPM, o $\beta$ representa a medida do risco sistemático, ou não-diversificável, da empresa em avaliação. Damodaran (2009, p.188) cita três formas disponíveis para estimar o parâmetro beta: através de dados históricos de preços de mercado para ativos específicos; a partir das características fundamentais de investimento e por meio de dados contábeis. Em seguida, o autor (2009, p.199) enumera três variáveis da empresa fundamentais na determinação de $\beta$ : tipo de negócio, grau de alavancagem operacional e grau de alavancagem financeira.

Quando se pretende comparar os betas das empresas no mercado, estimar o beta de uma empresa por meio de uma companhia similar no mercado ou identificar qual a participação do risco que está associado à alavancagem financeira ou à operação, Salmasi (2009) relata que se deve desalavancar o beta e alavancá-lo novamente de acordo com a estrutura de capital da empresa que se deseja avaliar. A equação utilizada para alavancar o beta é assim expressa:

$$
\begin{aligned}
& \beta_{\mathrm{L}}=\beta_{\mathrm{U}} \times\left(1+\frac{\mathrm{D}}{\mathrm{PL}}\right) \times(1-\mathrm{IR}) \\
& \text { Em que: } \\
& \beta_{\mathrm{L}}=\text { beta alavancado, determinado pelo nível de endividamento (D/PL); } \\
& \beta_{\mathrm{U}}=\text { beta não-alavancado (beta da empresa sem dívida); } \\
& \mathrm{D}=\text { dívida ou passivo oneroso; } \\
& \mathrm{PL}=\text { patrimônio líquido; } \\
& \mathrm{IR}=\text { alíquota de imposto de renda praticada pelas empresas brasileiras. }
\end{aligned}
$$

Damodaran $\left(2009\right.$, p. 201) traz a equação (7) utilizada na obtenção do beta desalavancado ( $\left.\beta_{U}\right)$ :

$$
\beta_{\mathrm{U}}=\beta_{\text {CORRENTE }} / 1+(1-\mathrm{IR})
$$

Considerando as peculiaridades de um país emergente, como o Brasil, Assaf Neto, Lima e Araújo (2008) propõem as seguintes etapas para se chegar ao coeficiente beta: (i) identificação do setor de atuação da empresa; (ii) levantamento do $\beta_{U}$ médio das empresas identificadas com a empresa (ou setor); (iii) cálculo do índice médio de endividamento do setor empresarial brasileiro para o qual deseja apurar o custo de capital; e (iv) com base nesse quociente, alavancar o beta através da equação 6 .

\subsubsection{Prêmio de risco de mercado - $(\mathbf{R m}-\mathbf{R f})$}

A taxa de prêmio relativa ao risco de mercado, também conhecida como ágio pelo risco de mercado, é obtida a partir da diferença entre a taxa de retorno esperada sobre a carteira do mercado (Rm) e a taxa livre de risco (Rf). Segundo Assaf Neto, Lima e Araújo (2008) a carteira de mercado, de acordo com a teoria do CAPM, deveria incluir todos os ativos negociados, 
ponderados pelos seus respectivos valores de mercado. Na prática a carteira de mercado contempla o retorno médio das ações negociadas (ativos com risco), sendo seu desempenho formal representado pelo índice de mercado de bolsa de valores.

A escolha da proxy da carteira de mercado é uma crítica tarefa. Pereiro (2002, p.118) relata que a principal referência no mercado acionário norte-americano é o Standard \& Poor's (S \& P) 500 Index, correspondente à ponderação das 500 ações mais negociadas na Bolsa de Nova Iorque (NYSE). No Brasil, o índice mais utilizado como proxy é o Índice da Bolsa de Valores de São Paulo (IBOVESPA).

Segundo Assaf Neto (2003) existem duas distintas abordagens para se estimar o prêmio pelo risco de mercado. A primeira abordagem considera o comportamento histórico das taxas dos ativos de risco (ações) em relação aos investimentos classificados como livres de risco (títulos públicos). Uma abordagem alternativa é determinar esse prêmio de acordo com a prática que os mercados financeiros vêm atualmente adotando de utilizar um mercado mais estável e de risco mínimo como referência.

A abordagem de prêmios históricos continua sendo a abordagem-padrão, a qual pode produzir razoáveis estimativas em mercados como os Estados Unidos, com uma bolsa de ações grande e diversificada e uma longa história de retornos tanto para ações como para títulos do governo, mas pode ser fracassada em países emergentes. Os principais aspectos desta abordagem são o período de tempo utilizado nas estimativas, a escolha do título livre de risco e o procedimento para estimativa do prêmio envolvendo a escolha entre a média aritmética e geométrica (DAMODARAN, 2009, p.165-166).

Pereiro (2002, p.119) coloca que o comprimento da série temporal utilizada resulta de um dilema entre tentar capturar o máximo de informações possíveis (período histórico mais longo) e, capturar os últimos acontecimentos os quais podem afetar radicalmente os retornos futuros (períodos mais curtos). Damodaran (1999) defende que o prêmio de mercado seja fundamentado em históricos mais longos e ressalta que em países emergentes, os quais, frequentemente, têm uma história confiável de 10 anos ou menos e desvios padrões grandes nos retornos anuais das ações, os prêmios de risco históricos não devem ser utilizados.

A taxa livre de risco escolhida para cálculo do prêmio de mercado deve ser a mesma do cálculo do retorno esperado. Segundo Pereiro (2002, p.119) autores divergem, contudo, na utilização da média aritmética ou da geométrica. Damodaran (1999), por exemplo, argumenta a favor da média geométrica por ser um melhor indicador de retorno a longo prazo e não superestimar o prêmio. Já Copeland, Koller e Murrin (2002, p.223) defendem a utilização da média aritmética, porque apesar de a média geométrica expressar adequadamente o retorno histórico, o CAPM é baseado em retornos esperados, o que é expresso de forma mais apropriada pela média aritmética dos retornos históricos.

\subsubsection{Prêmio de risco-país - $\alpha B R$}

De acordo com Pereiro (2006) o prêmio de risco-país é um agregado de riscos não sistemáticos, tais como: o risco derivado de instabilidade social e política o risco de inadimplemento soberano e risco de uma hiperinflação inesperada.

Para estimar o prêmio de risco-país, Damodaran (2009, p.170) aponta duas abordagens: a primeira explora o spread de inadimplência sobre bônus emitidos por cada país, que mede a diferença entre os bônus em dólar emitidos pelo país e a taxa do bônus do Tesouro americano; a segunda utiliza a volatilidade dos mercados de ações como base.

A liquidez e a maturidade do título C-Bond (capitalization bond), título brasileiro da dívida pública externa transacionado livremente no mercado internacional, mostram que ele é

R. Cont. Ufba, Salvador-Ba, v. 7, n. 3, p. 05 - 22, dezembro-março 2014 
representativo dos demais títulos soberanos do Brasil, sendo o mais utilizado para cálculo do prêmio pelo risco-país (MOREIRA e ROCHA, 2003).

Damodaran (2003) esclarece que dentre as diferentes medidas de risco-país, uma das mais simples e acessíveis é o rating (classificação orientada) atribuído à dívida do país por agências como a Standard \& Poor's (S \& P). Assaf Neto e Lima (2009, p. 507) apontam o indicador EMBI+ (Emerging Markets Bond Index Plus) como representativo do grau de risco-país classificado pelo banco de investimento J. P. Morgan, pioneiro nestas classificações, sendo bastante utilizado para economias emergentes como a do Brasil.

Outras possibilidades de mensuração do risco-país, elucidadas por Damodaran (2003), são medidas pela volatilidade dos mercados. Uma medida convencional do risco das ações é através do desvio-padrão nos preços das ações. A outra medida apontada pelo autor é baseada na expectativa de que o prêmio de risco de ativos de patrimônio do país seja maior que o spread de risco de inadimplência, e que tal prêmio aumentará se a classificação do país cair ou a volatilidade relativa do mercado de ações subir.

Damodaran (2003) resume que as três abordagens para estimar os prêmios de risco-país, em geral dar-lhes-ão resultados diferentes. Acredita que os maiores prêmios de risco-país (abordagem mista, spread de inadimplência + desvio padrão relativos) sejam mais realistas para o futuro imediato, mas com o passar dos anos os mesmos cairão e o prêmio de risco convergirá para o spread de bônus do país.

Assaf Neto (2003) discorre que no Brasil, geralmente, utiliza-se no modelo do CAPM o prêmio pelo risco de mercado dos EUA acrescido do $\alpha \mathrm{BR}$, apurado pela diferença entre as taxas de remuneração do bônus do governo norte-americano ( $T$-Bond) e o bônus do governo brasileiro $(C$-Bond $)$. A expressão 8 evidencia que o investidor demanda uma taxa de retorno superior ao que exigiria em um mercado de risco mínimo, como o dos EUA:

$$
\mathrm{Ke}=[\mathrm{Rf}+\beta \times(\mathrm{Rm}-\mathrm{Rf})]+\alpha \mathrm{BR}
$$

em que $\alpha$ BR representa o risco-Brasil.

Damodaran (2003) enfatiza que nem todas as empresas em mercados emergentes estão igualmente expostas ao risco-país, sendo necessário diferenciá-lo entre as empresas e que a exposição de uma empresa ao risco-país vem de onde ela faz seus negócios. O próprio autor (2009, p.211) discorre que a exposição de uma empresa ao risco-país é afetada por aspectos de sua atividade (como a localização de suas instalações). $\mathrm{O}$ autor aponta a análise de receitas como uma das maneiras mais simples de se estimar essa exposição:

$$
\lambda=\frac{\text { Proporção das receitas no país empresa }}{\text { Proporção das receitas no país empresa média }}
$$

onde lambda $(\lambda)$ é uma medida de exposição da empresa ao risco-país encontrada usando-se a proporção das receitas geradas em um país comparadas à proporção das receitas geradas pela média das empresas naquele país. Desta forma Damodaran $(2009$, p.175) chega à abordagem preferida por ele na busca de uma estimativa do custo do capital próprio para uma empresa brasileira, fundamentada em dados do mercado norte-americano e adaptada para que cada empresa tenha uma exposição ao risco-país $(\lambda)$ que seja diferente de sua exposição a todos os outros riscos de mercado:

$$
\mathrm{Ke}=\mathrm{Rf}+\beta \times(\mathrm{Rm}-\mathrm{Rf})+\lambda(\alpha \mathrm{BR})
$$


Damodaran (2003) salienta que embora a maioria concorde que há mais risco em investir em ações do Brasil que em ações norte americanas isto não é o suficiente para determinar a existência de um prêmio de risco adicional cobrado ao se investir no mercado brasileiro. A questão é se o risco do mercado emergente é ou não diversificável. Em mercados completamente integrados o investidor é diversificado globalmente e tais riscos são eliminados via diversificação. Em mercados completamente segmentados, no qual os investidores não investem fora do mercado local, adaptar o prêmio pelo risco-país também seria desnecessário. Ao passo que apenas em mercados parcialmente segmentados, estes riscos seriam relevantes na determinação do Ke.

Pereiro (2002, p.115) defende o uso de um CAPM Global (G-CAPM), representado pela expressão 11, em mercados fortemente integrados, e a utilização de um CAPM Local (LCAPM) quando o mercado doméstico está parcialmente ou não integrado com o mercado mundial, de forma a capturar os riscos específicos destes mercados.

$$
\operatorname{Ke}(\mathrm{G}-\mathrm{CAPM})=\mathrm{RF}_{\text {Global }}+\beta_{\text {Global }}\left(\mathrm{RM}_{\text {Global }}-\mathrm{RF}_{\text {Global }}\right)
$$

No L-CAPM, segundo o mesmo autor (2006), $\alpha$ BR é geralmente calculado como diferença entre a rentabilidade de títulos soberanos do mercado emergente denominados em dólar em relação aos títulos semelhantes de mercados desenvolvidos, por exemplo, T-bonds, se o mercado dos EUA for a proxy do mercado global. As críticas em torno da utilização da expressão 12 decorrem da inclusão do prêmio pelo risco de inadimplência na formação da $\mathrm{Rf}$ local $\left(\mathrm{RF}_{\text {Global }}+\alpha \mathrm{BR}\right)$, evidenciando uma tendência a superestimar o custo de capital, uma vez que o prêmio pelo risco de mercado já refletiria uma parcela deste risco.

$$
\operatorname{Ke}(\mathrm{L}-\mathrm{CAPM})=\left(\mathrm{RF}_{\text {Global }}+\alpha \mathrm{BR}\right)+\beta_{\text {Local }}\left(\mathrm{RM}_{\text {Local }}-\mathrm{RF}_{\text {Local }}\right)
$$

Ainda sobre o assunto, Copeland, Koller e Murrin (2002, p. 395) alertam sobre este perigo de dupla contagem do risco que ronda as avaliações de empresas. Damodaran (2009, p. 172) comenta casos em que os analistas utilizam como taxa livre de risco os bônus emitidos em dólar pelo país (por exemplo, o $C$-Bond no Brasil), cuja taxa de juros já incorpora os spreads de inadimplência e, ainda assim, ajustam um prêmio de risco a fim de refletir o risco-país, configurando então uma dupla contagem.

Pequenas empresas, em termos de valor de mercado do patrimônio líquido, obtêm retornos maiores que grandes empresas de risco equivalente (definido pelo beta de mercado). O porte do prêmio da pequena empresa (small cap premium) abaixou consideravelmente nos anos 90 mas ainda tem sido geralmente positivo e constitui uma medida da falha do beta em capturar o risco no CAPM, pois neste modelo os betas subestimam o verdadeiro risco das ações de pequenas empresas (Damodaran, 2009, p.139-140).

O mesmo autor (2009, p.214-215) reforça que alguns analistas defendem o acréscimo de um prêmio ao Ke de pequenas empresas, conforme a expressão 13 , e considera que $2 \%$ seja um valor razoável para tal parâmetro uma vez que, nas últimas décadas, a rentabilidade das empresas pequenas tem sido aproximadamente $2 \%$ maior do que a das grandes:

$$
\mathrm{Ke}=\mathrm{RF}+\beta \times \mathrm{PM}+\mathrm{SCP}
$$

sendo o $\mathrm{PM}=$ prêmio de risco de mercado $(\mathrm{Rm}-\mathrm{Rf})$ e o $\mathrm{SCP}=$ small cap premium ou prêmio pelo pequeno tamanho da entidade. 


\section{METODOLOGIA}

Com intuito de atingir o propósito desta pesquisa optou-se por um estudo exploratório e descritivo, uma vez que, na perspectiva do que defende Gil (2002, p.41), pretende possibilitar novas percepções sobre o tema, descrever situações presentes com dados colhidos da realidade, visando aclarar características do fenômeno em uma determinada população.

Quanto ao procedimento metodológico adotado para coleta de dados esta pesquisa é classificada como bibliográfica e documental, utilizando-se no exame de documentos, os Laudos de Avaliação das Companhias os quais são apresentados como requisito às ofertas públicas de aquisição de ações - OPA. Portanto, esses laudos de avaliação constituem meios para realização da pesquisa. Sua elaboração, estabelecida pela Lei 6.404/76, é normatizada pela Instrução da CVM 361/02 a qual, em seu Artigo $8^{\circ} \S 3^{\circ}$, fixa que o laudo deverá expressar o valor econômico da companhia objeto por ação, calculado pela regra do fluxo de caixa descontado ou por múltiplos.

Por questões de acessibilidade, foram utilizadas as informações públicas obtidas da Comissão de Valores Mobiliários. Foram selecionados os laudos de avaliação no período compreendido de 2005 e 2012, desprezando-se aqueles que não apuraram o valor econômico da empresa pelo método do fluxo de caixa descontado. Após a delimitação o universo da pesquisa perfez 63 laudos de avaliação cujas empresas estão relacionadas no Anexo I.

Para viabilizar a coleta de dados, optou-se nesta pesquisa pelo uso do formulário. Constam do formulário 18 perguntas categorizadas (A-R) as quais foram elaboradas a partir da teoria levantada na seção 2. Para cada interrogativa foram sugeridas respostas fundamentadas também na literatura pesquisada, a fim de possibilitar a verificação da aderência da prática à teoria. As respostas foram enumeradas, sendo que a última alternativa de resposta para todas as interrogações é a opção "não evidenciada". Definidas as perguntas, foram investigados criteriosamente os 96 laudos.

Os dados obtidos foram tabulados e expostos em uma tabela de forma sintética. Foram somadas as quantidades de cada resposta recorrentes em cada pergunta, em seguida estas quantidades foram convertidas em percentuais após relacioná-las com a quantidade total de laudos utilizados na extração das respostas para as respectivas perguntas.

Durante a redação da análise dos resultados, os percentuais retirados das tabelas serão acompanhados da identificação da pergunta (letras do alfabeto) e respostas (números que podem variar de 1 a 6 ). Após a coleta de dados, todas as informações reunidas nas tabelas são comparadas entre si e confrontadas com a teoria, com vistas à rejeição ou não da hipótese.

\section{RESULTADOS}

O Quadro 1 apresenta os resultados das questões formuladas condizentes a teoria levantada, sobre o custo do capital próprio na avaliação de empresas.

Quadro 1 - Custo do Capital Próprio

\begin{tabular}{|clr|}
\hline \multicolumn{2}{|l|}{ Resultados da Pesquisa - Custo do Capital Próprio } & Laudos (\%) \\
\hline A) & Ao utilizar o FCD, qual Fluxo de Caixa escolhido? & $79,17 \%$ \\
A1 & FCLE & $17,71 \%$ \\
A2 & FCLA &
\end{tabular}

R. Cont. Ufba, Salvador-Ba, v. 7, n. 3, p. 05 - 22, dezembro-março 2014 


\begin{tabular}{|rlr|} 
A3 & Outros (1 e 2) & $1,04 \%$ \\
A4 & Não evidenciado & $2,08 \%$ \\
\hline B) & Na escolha do FCLE quantos usaram & \\
B1 & WACC & $90,79 \%$ \\
B2 & Ke & $7,89 \%$ \\
B3 & Kd & $1,32 \%$ \\
B4 & Não evidenciado & $0,00 \%$ \\
\hline C) & No caso do FCLA quantos usaram & \\
C1 & WACC & $5,88 \%$ \\
C2 & Ke & $94,12 \%$ \\
C3 & Kd & $0,00 \%$ \\
C4 & Não evidenciado & $0,00 \%$ \\
\hline D) & Metodologias aplicadas na mensuração do Ke: & \\
D1 & Modelo de Gordon & $0,00 \%$ \\
D2 & Modelo Ohlson-Juettner (OJ) & $0,00 \%$ \\
D3 & Arbitrage Princing Model (APM) & $0,00 \%$ \\
D4 & Capital Asset Pricing Model (CAPM). & $100,00 \%$ \\
D5 & Método de Prêmios de Risco (Build-up Models) & $0,00 \%$ \\
D6 & Não evidenciado & $0,00 \%$ \\
\hline E) & No CAPM, adota taxa livre de risco (Rf): & \\
E1 & Rf Local = SELIC & $2,11 \%$ \\
E2 & Rf Local = RF Global + $\alpha$ BR & $16,84 \%$ \\
E3 & Rf Global & $71,58 \%$ \\
E4 & Outros (Exemplo: Rf local = poupança) & $3,16 \%$ \\
E5 & Não evidenciado & $6,32 \%$ \\
\hline F & Os & \\
\hline
\end{tabular}

F) Os instrumentos utilizados para a obtenção da Rf Global nos EUA:

F1 T-bill (letras do Tesouro de curto prazo)

F2 T-bonds 10 anos

F3 T-bonds 30 anos

F4 Outros

F5 Não evidenciado

$10,00 \%$

G) O $\beta$ usado no CAPM foi estimado a partir de:

G1 dados históricos de preços de mercado para ativos específicos

G2 características fundamentais de investimento

G3 dados contábeis

G4 Outros

G5 Não evidenciado

H) $\mathbf{O} \boldsymbol{\beta}$ escolhido foi:

H1 $\beta \mathrm{LL}=$ Beta local em relação ao índice do mercado local

H2 $\beta \mathrm{LG}=$ Beta local em relação ao índice do mercado global

H3 Outros

I) Quanto ao $\beta$ lançado no CAPM:

I1 $\beta$ alavancado 


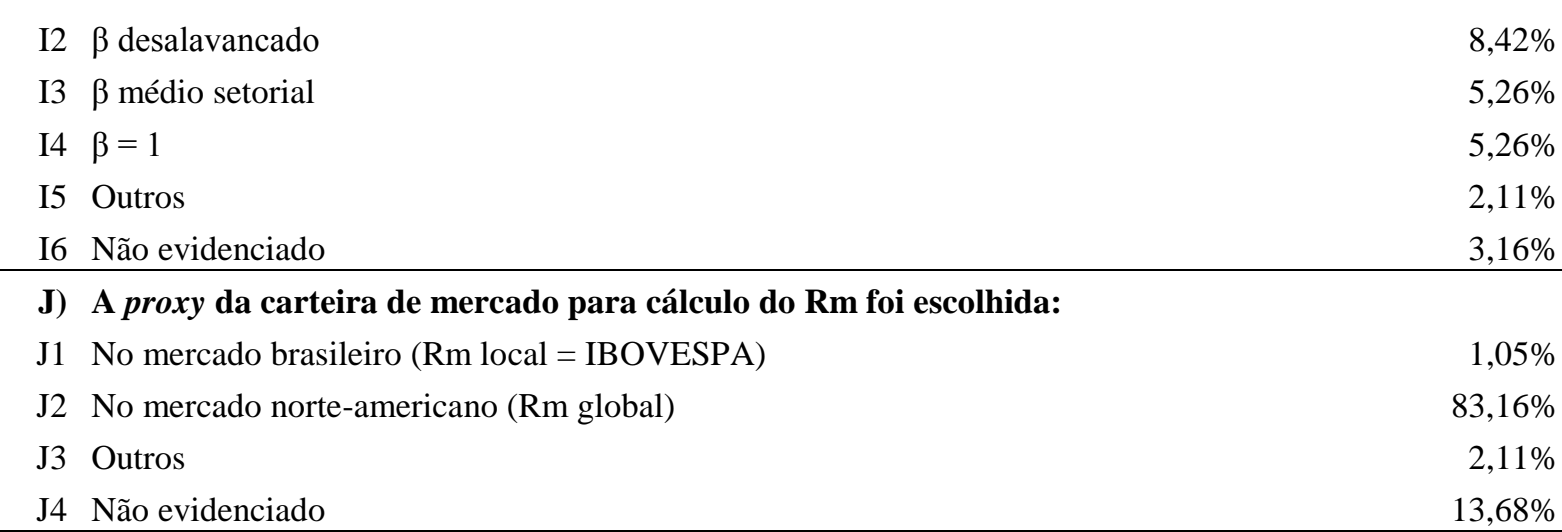

K) O Rm Global utilizado foi com base nos retornos históricos:

$\begin{array}{llr}\text { K1 Standard \& Poor's (S \& P) } 500 \text { Index da NYSE } & 25,81 \%\end{array}$

\begin{tabular}{ll} 
K2 Outros & $7,53 \%$ \\
\hline &
\end{tabular}

\begin{tabular}{ll} 
K3 Não evidenciado & $66,67 \%$ \\
\hline
\end{tabular}

L) O comprimento da série temporal escolhida no cálculo do Rm está:

$\begin{array}{ll}\text { L1 Abaixo de } 10 \text { anos } & 0,00 \%\end{array}$

$\begin{array}{lr}\text { L2 Entre } 10 \text { e } 20 \text { anos } & 3,16 \%\end{array}$

$\begin{array}{ll}\text { L3 Acima de } 20 \text { anos } & 50,53 \%\end{array}$

$\begin{array}{lr}\text { L4 Não evidenciado } & 46,32 \%\end{array}$

M) A Rf escolhida para cálculo do Pm é a mesma do cálculo Ke

M1 Sim

M2 Não

M3 Não evidenciado

N) A média dos retornos históricos aplicada na mensuração do Pm foi:

N1 Média aritmética

N2 Média geométrica

N3 Outras

N4 Não evidenciado

O) Adicionaram o $\alpha B R$ ao CAPM?

O1 Sim

O2 Não

O3 Não evidenciado

P) Para determinação do $\alpha \mathrm{BR}$ qual a abordagem utilizada?

P1 Spread de inadimplência sobre bônus emitidos por cada país

P2 Abordagem do desvio-padrão relativo

P3 Abordagem mista: spread de inadimplência + desvio padrão relativos

P4 Outras

P5 Não evidenciada

Q) Dos que calcularam $\alpha B R$, quantos:

Q1 Consideraram $\lambda=$ exposição específica da empresa ao $\alpha \mathrm{BR}$

Q2 Não consideraram $\lambda$

R) Além do $\alpha B R$, quantos adicionaram outros prêmios ao CAPM como:

R1 Small cap premium (prêmio pelo tamanho da empresa) 
R2 Outros prêmios

R3 Não adicionaram

R4 Não evidenciado

Fonte: Elaborado pelos autores

Dentre as abordagens existentes no uso do FCD destacam-se: o FCLE - Fluxo de Caixa Livre da Empresa e o FCLA - Fluxo de Caixa Livre do Acionista. Assaf Neto e Lima (2009, p.740) e Copeland, Koller e Murrin (2002, p.137) consideraram que a preferência pelo FCLE se deve ao fato de o modelo promover uma maior evidenciação de informações, já que engloba todos os componentes da empresa (capital próprio e de terceiros).

De forma coerente com o apontamento teórico a investigação realizada traz que a maior parte dos avaliadores optou pelo FCLE (A1), dos quais 90,79\% usaram o WACC para descontar tais fluxos (B1). A aderência entre a taxa de desconto e o fluxo de caixa também é percebida na maioria dos laudos que optaram pelo FCLA (C2).

O destaque dado ao FCLE e ao FCLA também pôde ser confirmado na prática dos avaliadores brasileiros. Dentre os laudos que evidenciaram o método do FCD aplicado na mensuração do valor da empresa, todos utilizaram os dois fluxos mencionados, seja isoladamente (A1 e A2), ou através da utilização simultânea de ambos no mesmo laudo (A3).

Nos laudos analisados observou-se a mesma popularidade do modelo CAPM que fora referenciada na teoria, já que $100 \%$ (D4) dos laudos que calcularam o custo do capital próprio o fizeram através deste modelo. Diante do ocorrido todos os outros parâmetros apresentados na Tabela são componentes do CAPM, os quais foram descritos no item 2.2 deste artigo.

Quanto à Taxa Livre de Risco - Rf nota-se a predominância do uso de benchmark de economias desenvolvidas, principalmente dos EUA. Esta prática é recorrente nos demais componentes do CAPM, conforme preconizado pela literatura. A Selic, parâmetro brasileiro para ativo livre de risco, aparece em apenas 2,11\% (E1) dos laudos. Por outro lado, aproximadamente $88 \%$ (E2 + E3) dos analistas usaram o Rf Global para determinar a taxa livre de risco a ser aplicada no CAPM, ao qual 16,84\% (E2) já adicionaram o risco-país a esta Rf Global.

As três principais opções de Rf nos EUA foram destacadas na interrogativa "F" da Tabela. A literatura pesquisada recomenda a taxa dos títulos do T-bonds de 10 anos por motivos anteriormente discutidos. A prática demonstra que $40 \%$ (F2) dos laudos, ou seja, a maioria adere a esta preferência teórica.

Dentre as três diferentes formas existentes para se estimar o $\beta$ as quais foram citadas por Damodaran (2009, p. 188), 86,32\% (G1) optaram por apurá-lo com dados históricos de preços de mercado para ativos específicos, através da média de betas de empresas representativas do setor. A análise dos resultados possibilita outras constatações sobre o beta lançado no CAPM: mais de $70 \%$ (H2) calcularam o $\beta_{\mathrm{LG}}$, o qual corresponde a um beta local em relação ao índice do mercado global; 75,79\% (I1) usaram-no após alavancá-lo obedecendo à estrutura de capital da empresa.

A proxy da carteira de mercado para cálculo do $\mathrm{Rm}$ foi escolhida em sua evidente maioria nos mercados desenvolvidos, pois apenas $1,05 \%$ (J1) utilizaram o índice da BOVESPA, enquanto 83,16\% (J2) dos laudos calcularam o Rm Global. A referência norte-americana apontada pela literatura como principal na determinação do Rm Global é o Standard \& Poor's (S \& P) 500 Index. Examinando-se os laudos percebe-se que 66,67\% (K3) não demonstraram qual a referência global adotada e, dentre os que a evidenciaram, 25,81\% (K1) fizeram uso do S\&P 500 .

O período de tempo utilizado nas estimativas do Rm foi outro assunto abordado no referencial teórico. Verifica-se que a prática dos avaliadores neste quesito é condizente com a literatura, 
visto que a mesma defende a utilização de períodos históricos mais longos. Conforme verificado na Tabela, 53,69\% (L2 + L3) informaram o comprimento da série temporal escolhida, destes, $3,16 \%$ (L2) capturaram períodos entre 10 e 20 anos e 50,53\% (L3) optaram por períodos maiores que 20 anos, vale ressaltar que, os órgãos regulatórios de concessões públicas exigem as projeções de fluxos de caixa para avaliação, dentro do período de concessão, que no Brasil gira-se em torno de 30 anos.

Dando prosseguimento à análise do Prêmio de Mercado, 68,42\% (M3) não mencionaram qual a taxa livre de risco utilizada, porém verifica-se que praticamente $25 \%$ (M1) utilizaram a mesma Rf do cálculo do Ke. Percebe-se ainda um baixo índice de evidenciação, 17,90\% (N1+ N2), da média dos retornos históricos aplicada na mensuração do Pm, se aritmética - 11,58\% (N1) ou geométrica $-6,32 \%(\mathrm{~N} 2)$. Os resultados obtidos na interrogativa "N" pode ser um reflexo da divergência de opiniões dos próprios teóricos.

$\mathrm{O}$ acréscimo de um prêmio pelo risco inerente ao país é consideravelmente defendido, conforme exposto na seção 2. Constata-se que nas avaliações brasileiras que foram exploradas nesta pesquisa, 91,58\% (O1) adicionaram o risco-Brasil - $\alpha \mathrm{BR}$ ao CAPM, 7,37\% (O3) não o evidenciaram, e apenas $1,05 \%(\mathrm{O} 2)$ dos laudos não calcularam este prêmio.

Quanto à abordagem apontada para determinação do $\alpha \mathrm{BR}$, desprezando-se os 22,22\% (P5) que não informaram, todos os outros 75,56\% (P1) afirmaram ter explorado o spread de inadimplência, através da diferença entre os bônus em dólar emitidos pelo país e a taxa do bônus do Tesouro americano. A medida de exposição ao risco-país - $\lambda$, tão significativa para Damodaran (2009, p. 175), não foi verificada em nenhum dos laudos: 91,11\% (Q2) não consideraram e os restantes, 8,89\% (Q3), não evidenciaram.

Em 21,06\% (R1+R2) dos laudos que calcularam Ke surgiram outros prêmios adicionados ao modelo CAPM (que não o prêmio pelo risco-país), dos quais $18,95 \%$ (R1) são os conhecidos "prêmio pelo pequeno tamanho da empresa", e 2,11\% utilizaram-se de outros prêmios (R2). Configura-se em "outros prêmios" o prêmio pela falta de liquidez cuja aplicação não fora apontada dentre os autores pesquisados. Já o small cap premium ou prêmio pelo tamanho da empresa foi mencionado como adequado em certas conjunturas.

Diante do exposto e análises dos dados coletados da realidade à teoria exposta no capítulo 2 , considera-se a não rejeição da hipótese proposta por esta pesquisa. A confirmação da $\mathrm{H}$ é factível já que as premissas acerca do cálculo do Ke caracterizadas pelos autores em finanças foram, em maior percentual, atendidas pelos analistas responsáveis pela elaboração dos laudos.

\section{CONSIDERAÇÕES FINAIS}

A prática da avaliação de empresas se difundiu significativamente no Brasil nas últimas décadas. As informações geradas por esta ferramenta são utilizadas por uma considerável gama de usuários. A importância do tema provoca teóricos em finanças, praticantes do mercado, a academia e também se configurou como motivadora desta pesquisa.

Dentre as metodologias existentes para mensuração do valor econômico das entidades, destacase o método do Fluxo de Caixa Descontado cujo sucesso depende da correta determinação da taxa de desconto no qual a mensuração do Ke tem grande destaque. Neste contexto, o presente estudo almejou verificar no Brasil se as premissas adotadas pelo mercado no processo de estimação desta taxa estão condizentes com a teoria.

A fim de atingir seu objetivo, este trabalho, de caráter descritivo e exploratório, propôs uma pesquisa bibliográfica e qualitativa concomitante e comparativamente a uma pesquisa 
documental, investigando-se os laudos de ofertas públicas de ações - OPAs dos anos de 2005 a 2012.

A análise de resultados comprovou a aderência dos métodos utilizados pelos praticantes brasileiros e a literatura de finanças sobre o tema, levando a não rejeição da hipótese proposta inicialmente. Desta forma, as evidências encontradas neste estudo contribuem com os estudiosos, avaliadores e demais interessados no assunto e, atribui-se maior credibilidade às informações geradas pelas avaliações de empresas no Brasil, qualitativamente.

Podem ser vislumbradas, a partir deste estudo, outras indagações as quais sugerem pesquisas posteriores, tais como: (i) investigação de aderência da prática e teoria na mensuração das demais taxas de desconto utilizadas no método do FCD (WACC e custo da dívida - Kd); (ii) necessidade de pesquisas quantitativas para relacionar o projetado nessas taxas e o realizado; (iii) aumento da amostra, com inclusão de IPOs (Initial Public Offering), por exemplo.

\section{REFERENCIAS}

ASSAF NETO, Alexandre. Contribuição ao estudo de avaliação de empresas no Brasil: Uma aplicação prática. 203p. Tese (Livre-Docência) - Departamento de Contabilidade de Faculdade de Economia, Administração e Contabilidade de Ribeirão Preto, São Paulo: Universidade de São Paulo, 2003.

ASSAF NETO, Alexandre; LIMA, Fabiano Guasti. Curso de Administração Financeira. São Paulo: Atlas, 2009

ASSAF NETO, Alexandre; LIMA, Fabiano Guasti; ARAUJO, Adriana Maria Procópio de. Uma proposta metodológica para o cálculo do custo de capital no Brasil. Revista de Administração, São Paulo, v.32, n. 1, p.72-83, jan./fev./mar.2008.

COMISSÃO DE VALORES MOBILIÁRIOS (CVM) - Instrução Normativa 361/2002. Disponível em: www.cvm.gov.br. Acesso em 02 de maio de 2010.

COPELAND, Tom; KOLLER, Tim; MURRIN, Jack. Avaliação de Empresas - Valuation: Calculando e gerenciando o valor das empresas. Tradução: Allan Vidigal Hastings. 3. ed. São Paulo: Makron Books, 2002.

DAMODARAN, Aswath. Avaliação de investimentos: ferramentas e técnicas para a determinação do valor de qualquer ativo. 2. ed. Rio de Janeiro: Qualitymark, 2009.

DAMODARAN, Aswath. Estimating Equity Risk Premiums. Stern School of Business. 24p. New York, 1999 (Working Paper). Disponível em: <http://www.stern.nyu.edu/fin/work papers/papers99/wpa99021.pdf> Acesso em 07 abr. 2010.

DAMODARAN, Aswath. Measuring Company Exposure to Country Risk: Theory and Practice. Stern School of Business. 30p. New York, 2003 (Working Paper). Disponível em: <http://papers.ssrn.com/sol3/papers.cfm?abstract_id=889388> Acesso em 07 abr. 2010.

FERNÁNDEZ, Pablo. Company Valuation Methods: The Most Common Errors in Valuations. PricewaterhouseCoopers Professor of Corporate Finance. 30p. Madrid. IESE 
Business School, 2007. Disponível em: < http://www.iese.edu/research/pdfs/DI-0449-E.pdf>. Acesso em 03 mar. 2010.

GARRÁN, Felipe Turbuk; MARTELANC, Roy. Metodologias em uso no Brasil para Determinação do Custo de Capital Próprio. In: ENCONTRO DA ANPAD, XXXI, 2007, Rio de Janeiro. Anais... Rio de Janeiro: EnANPAD, 2007. 1 CD ROM.

GIL, Antônio Carlos. Como elaborar projetos de pesquisa. 4. ed. São Paulo: Atlas, 2002.

LIMA, Gerlando Augusto Sampaio Franco de. Utilização da teoria da divulgação para avaliação da relação do nível de disclosure com o custo da dívida das empresas brasileiras. 108p. Tese (Doutorado em Controladoria e Contabilidade) - Departamento de Contabilidade e Atuária FEA/USP. São Paulo: Universidade de São Paulo, 2007.

MARTINS, Eliseu; GALDI, Fernando Caio; LIMA, Gerlando Augusto Sampaio Franco de; NECYK, George Anthony; ABE, Cesar Henrique Shogi. Evidências Empíricas de Modelos de Estimação do Custo do Capital Próprio. BBR - Brazilian Business Review, Vitória, v. 3, n. 2, p. 137-156, jul./dez. 2006.

MOREIRA, Ajax R. B.;ROCHA, Kátia. Determinantes do Risco Brasil: Fundamentos e Expectativas - Uma abordagem de Modelos de Risco de Crédito. IPEA - Texto para Discussão, n. 945, Rio de Janeiro, Abril de 2003. Disponível em: 〈http://www.ipea.gov.br〉 Acesso em 04 ago. 2010.

PEREIRO, Luis E. Valuation of Companies in Emerging Markets: A Pratical Approach. New York, John Wiley \& Sons, 2002.

PEREIRO, Luis E. The practice of investment valuation in emerging markets: Evidence from Argentina. Journal of Multinational Financial Management. v. 16, n. 2, p.160-183, abr. 2006.

SALMASI, Silvia Vidal. Estudo sobre os Fatores Determinantes do CAPM no Brasil. In: Seminário de Administração - SemeAD, 12, São Paulo, 2009. Anais... São Paulo: FEA/USP/Programa de Pós-Graduação em Administração, 2009. 1 CD-ROM. 
ANEXO I

\begin{tabular}{|c|c|}
\hline \multicolumn{2}{|c|}{ Laudos } \\
\hline AES Sul Distribuidor Gaúcha De Energia S.A & Fertilizantes Fosfatados S.A. Fosfertil \\
\hline Abyara Planejamento Imobiliário S.A & Globex Utilidades S.A \\
\hline Acesita S.A & Granóleo S/A \\
\hline Aços Villares S.A & GVT Holding \\
\hline AGF Brasil Seguros S.A & Indústrias Arteb S.A \\
\hline ALL - América Latina Log. Malha Norte S.A. & Indústrias Micheletto S.A \\
\hline ALL, Ferrovias Bandeirantes S.A. - $1^{\circ}$ laudo & IronX Mineração S.A \\
\hline ALL, Ferrovias Bandeirantes S.A. - $2^{\circ}$ laudo & Jari Celulose S.A \\
\hline Ampla Energia e Serviços S.A. & JBS S.A. \\
\hline Ampla Investimentos e Serviços S.A. & Magnesita S.A \\
\hline Arcelor Brasil S.A & Manasa Madeireira Nacional S.A \\
\hline ArcelorMittal Inox Brasil S.A & Marisol Sa \\
\hline Bacraft S/A Indústria de Papel & Medial Saúde S.A \\
\hline Banco BERJ S.A. & Melpaper S.A \\
\hline Banco do Estado do Ceará S.A & Parmalat Brasil S.A Indústria de Alimentos \\
\hline Banex S.A Crédito Financ. e Investimento & Perdigão S.A. e Eleva Alimentos S.A \\
\hline BANRISUL S.A. Administradora De Consórcios & PetroflexIndústria e Comércio S.A \\
\hline Calçados Azaléia S.A & Petroquímica União S.A \\
\hline Camargo Correa Desenv. Imobiliario S.A. & Plascar Participações Industriais S.A \\
\hline Centennial Asset Participações Sudeste S.A. & Pronor Petroquimica S.A. \\
\hline Cia Bandeirantes Arms Gerais & QGN Participações S.A. \\
\hline Cia de Seguros Minas Brasil & Redecard S.A. \\
\hline Cia Fabril Mascarenhas & Renner Participações S.A \\
\hline Cia Fluminense de Refrigerantes & Rimet Empreend. Inds. E Comerciais S.A. \\
\hline Cia Maranhense Refrigerantes & S.A. Fábrica de Produtos Alimentícios Vigor \\
\hline Cia Maranhese de Refrigerantes & Santista Têxtil S.A. \\
\hline Cia. Eldorado de Hoteis & Seara Alimentos S.A \\
\hline CMA - Participações S.A & SEB - Sistema Educacional Brasileiro S.A. \\
\hline Companhia Brasileira de Cartuchos & SEB Participações S.A \\
\hline Companhia de Bebidas das Américas - AmBev & SEB Participações S.A \\
\hline Companhia de Tecidos Santanense S.A & SEB Participações S.A \\
\hline Companhia de Trans. de E Elétrica Paulista S.A & Semp Toshiba S.A \\
\hline Companhia Iguaçu de Café Solúvel & Servix Engenharia S.A \\
\hline Companhia Leco de Produtos Alimentícios & Sola S.A. Inds Alimenticias \\
\hline Companhia Siderúrgica Paulista - Cosipa & Sul América Companhia Nacional de Seguros \\
\hline Confab Industrial S.A. & Suzano Petroquímica S.A \\
\hline Construtora Adolpho Lindenberg S.A. & TAM S.A. \\
\hline Copesul - Companhia Petroquímica do Sul & Technos Relógios S.A \\
\hline Cosan S.A & Tele Norte Celular Participações S.A. \\
\hline Dana-AlbarusS.A. Indústria e Comércio & Telemig Celular Part. S.A. / Telemig Celular S.A \\
\hline ECISA- Engenharia, Comércio e Indústria S/A & Terna Participaçoes \\
\hline Eletromoura S.A & Tivit Terceirização de Proc., Serv. e Tecnologia \\
\hline Embratel Participações S.A & Trafo Equipamentos Elétricos S.A \\
\hline Excelsior Alimentos S.A & Ultrapar Participações S.A \\
\hline FAE Adm. e Participações S.A. & Universo Online S.A. \\
\hline FAE- Ferragens Aparelhos Elétricos S.A. & Vale Fertilizantes S.A. \\
\hline Ferronorte S.A. - Ferrovias Norte Brasil & VIVO Participações S.A. \\
\hline
\end{tabular}

R. Cont. Ufba, Salvador-Ba, v. 7, n. 3, p. 05 - 22, dezembro-março 2014 
i 0 termo "livre" significa fluxo de caixa resultante depois de realizados: a) os investimentos necessários para a continuidade do negócio: investimentos em capital de giro e ativos fixos (este é o "livre" para a empresa ou dos detentores de capitais de terceiros e próprio), e b) após os fluxos de pagamentos para os proprietários de capitais de terceiros.

ii Em inglês: Weighted Avarege Cost of Capital. 